\title{
Une approche de la complexité en géographie
}

Denise Pumain

Professeur, Université Paris I

pumain@parisgeo.cnrs.fr

\begin{abstract}
A theory of geography should not be merely a spatial translation of theories of history or social change. It is suggested that concepts and methods derived from the theory of complex systems could be used in formalising the observations of the evolution of spatial systems. During the last thirty years, new developments of physical theories of selforganisation, evolution of living species and adaptive cognitive systems have changed our representations of system dynamics, especially about the conditions of emerging structures. The main epistemological questions focussed on the autonomy of systems, then on the identification of attractors governing their dynamics and lastly on their capacity to innovate. Geography could provide an interpretation of the genesis and evolution of localised structures, which are interposed at different levels between the persons and the world as are social networks or institutions.
\end{abstract}

$* * * * * * * * *$

Porter témoignage des influences philosophiques ou idéologiques en géographie, à partir de sa propre expérience, n'offre pas la meilleure garantie de l'objectivité scientifique! Décevoir Popper n'implique pas davantage de satisfaire les tenants d'une posture postmoderne, car, comme dit le proverbe russe : «le pire témoin est celui qui était là ». La proposition de la revue Géocarrefour et de François Durand-Dastès, tendant à une clarification des positions des géographes dans l'univers des références intellectuelles du temps, n'est cependant pas sans intérêt. Cette exigence d'explicitation, tout autant qu'un rappel de nos revendications affichées depuis les années 1970, invite aussi à un retour sur l'expérience des trente dernières années, du moins pour ce qu'il en reste de mémoire, avec les inévitables clairs-obscurs et révisions de sens qu'apporte le coup de projecteur des conceptions actuelles.

Dans cette optique, la question qui pour moi demeure centrale est celle des fondements d'une théorie de la géographie, qui serait une théorie permettant de comprendre l'évolution des systèmes territoriaux planétaires, ou plus précisément une théorie explicitant les processus (écologiques et spatiaux) qui conduisent à l'organisation et à la différenciation des entités localisables et des représentations que s'en font les sociétés. Il n'est pas possible d'évoquer en un court article les références correspondant à cette ambition théorique dans son ensemble, j'ai donc choisi d'en mentionner ici l'une des entrées possibles, qui n'est ni la seule, ni sans doute la principale, mais qui suscite aujourd'hui un regain de curiosité. J'ai ainsi l'occasion de répondre un peu longuement à la question qui m'a souvent été posée : pourquoi aller chercher une légitimité scientifique du côté des sciences « dures », pourquoi travailler en géographie avec des modèles ? Ma réponse sera construite autour de références à ce que l'on appelle aujourd'hui les théories de la complexité.

Les conditions de production d'un article imposent un biais d'écriture, j'ai choisi de ne mentionner ici que les références qui ont un rapport direct avec l'objet de mes recherches. Cela ne veut pas dire qu'il n'y en a pas eu d'autres, (dont éventuellement je n'aurais pas conscience !) et cela ne signifie pas non plus mon adhésion totale aux thèses de celles que j'ai citées. Si j'ai fait le choix personnel de ne pas militer pour une école, de garder un éclectisme 
de références, une liberté de construction intellectuelle, c'est qu'entre une posture philosophique qui tendrait à produire un système de pensée cohérent et une pratique des sciences sociales qui adopte l'insertion progressive et fragmentaire de faits d'observation dans des constructions interprétatives partielles, je me situe résolument dans la seconde voie. Cela n'implique pas une revendication d'originalité, puisque au contraire je crois à la nécessité d'une production et d'une transmission cumulatives de la connaissance. Cette position n'exclut pas les révisions nécessaires, ni l'ouverture, mais suppose une défiance à l'égard des concepts jetables, des acceptions personnelles et inusitées, et justifie au contraire le patient travail de réflexion et d'assimilation collective de la nouveauté.

\section{Théorie géographique et dynamique de système}

L'ambition théorique était très partagée, pour les géographes de ma génération, allant parfois jusqu'à proposer d'axiomatiser la géographie, ou du moins d'en approfondir les concepts et les construits (pour citer les titres de quelques-uns des premiers colloques Géopoint). Il est possible qu'elle ait été facilitée par les acquis taxinomiques antérieurs, par les nombreuses classifications de paysages, de régions, de genres de vie, de villes et de réseaux urbains, sans oublier les maisons et les peuples, les climats et les végétations, ou encore les formes de sousdéveloppement. Théoriser, c'était d'abord tenter d'échapper aux apories d'une " science du concret », et surtout dépasser l'irréductible unicité des lieux, chercher à construire un point de vue nomothétique sur la discipline.

Schématiquement, deux conceptions de la place de la théorie en géographie s'affrontaient à l'époque. Pour les uns, il était exclu de faire bande à part sous la bannière disciplinaire, toute construction spécifique revenant à "fétichiser l'espace», les formes et différenciations observées sur le terrain n'étant que la projection spatiale des organisations sociales, il était inutile (et forcément dangereux !) de chercher à identifier des effets de la composante spatiale sur les constructions sociales, donc une théorie géographique ne pouvait être d'abord qu'une théorie sociale. Pour d'autres, les régularités observées dans l'organisation de l'espace par les sociétés étaient suffisamment importantes, par delà les différences et inégalités des conditions « naturelles », politiques ou culturelles, pour en tenter la modélisation, voire un développement de théories comme celles déjà bien établies de la rente foncière de von Thünen sous-tendant les inégalités centre-périphérie à moyenne échelle, ou de la trame hiérarchisée des lieux centraux formalisée par Christaller.

Les jalons n'ont pas manqué dans cette quête d'une construction théorique plus assurée. Vues d'Europe, les questions posées par la géographie n'étaient pas mauvaises, le renouvellement ayant été amorcé par la génération précédente (la réévaluation de la hiérarchie des facteurs explicatifs s'y étant opérée sous la forme d'une ouverture thématique : meilleure prise en compte de l'économie, remise à sa place de la géomorphologie, remplacement du découpage zonal par la notion de développement). Rien d'étonnant donc si la « révolution théorique et quantitative» s'y est voulue plus en continuité qu'en rupture épistémologique (Racine, Reymond, 1973), et si l'accent a plutôt été mis sur la méthodologie, par exemple par l'incitation à ne pas se contenter de monographies (Pinchemel), au moment où la mise à disposition des techniques d'analyse multivariée et des premiers progiciels statistiques permettait de donner de la diversité géographique une description plus contrôlée, plus comparative, délaissant momentanément les perceptions intuitives et subjectives du paysage visible pour les certitudes partagées et rassurantes (mais en partie illusoires) des «paysages statistiques » (Pumain, Robic, 2002)! 
Ce n'est pas je crois refaire l'histoire que de signaler qu'à aucun moment nous n'avons souscrit à une interprétation des résultats de ces analyses, les facteurs récurrents et stables issus des analyses factorielles (par exemple, Pumain, Saint-Julien, 1978), en termes de « dimensions latentes », de «trames indépendantes » qui se seraient superposées selon une structure feuilletée de l'espace géographique (Lacoste, 1976), organisé par des logiques sectorielles. Ces interprétations, sans doute cohérentes avec la notion de «combinaison » de la géographie classique, et dont elles proposaient une forme de mesure, ne tenaient pas longtemps, dans les applications aux analyses comparatives des villes, face à une lecture en termes de traces d'une évolution. Les différenciations observées en termes de tailles des villes (et de tous les indicateurs qui lui sont associés) reflétaient l'histoire des effets cumulatifs de la croissance urbaine, tandis que les faisceaux d'indicateurs qualitatifs regroupés par différenciation fonctionnelle s'interprétaient en termes de cycles d'innovation et de "générations » de villes. Autrement dit, même si elles se traduisent par des configurations géographiques distinctes, les trames spatiales sont organisées par des processus temporels, et ce sont eux qui reproduisent ou maintiennent la stabilité de cette structure dans ses différentes composantes. L'explication de la pérennité de la combinaison ne réside pas tant dans la force des associations qui en relient les composantes, que dans la subtilité des principes évolutifs par lesquels la structure, de manière incrémentale et continue, et dans toutes ses composantes, s'adapte aux changements, qu'elle peut aussi d'ailleurs contribuer à susciter.

Très vite donc, cette quête théorique impliquait de savoir passer de l'observation des régularités à l'analyse des processus, de l'identification de « combinaisons » ou de structures, à l'étude du changement susceptible de les produire et de les transformer. Il faut préciser ce que l'on entend par changement. Bien souvent, la description du changement en géographie se limite à l'enregistrement des transformations politiques, techniques, économiques, sociales ou culturelles intervenant dans différents lieux. Il en résulte une géographie, certes nécessaire, que j'ai appelée « de la mise à jour » (Pumain, 1998). Dans une seconde étape, si l'on veut produire une théorie géographique du changement, il faut cependant déplacer la focale, depuis le contenu de ces transformations, vers les modalités géographiques de leur assimilation par les lieux, et de leurs effets en termes d'évolution des inégalités et des différenciations. Cela implique à la fois d'observer les transformations des structures des systèmes géographiques, en mesurant les effets différentiels et sélectifs du changement général, et de s'intéresser aux processus opérant ces différenciations. On oublie trop souvent que la New geography (comme l'avait bien vu Paul Claval, 1977), dans sa révolution, s'efforçait déjà d'expliquer les formes spatiales par des processus générateurs, rapportés à l'échelle des individus: catégories fondamentales des dispositions individuelles dans l'espace (Nystuen et l'exemple de la mosquée), préférences et représentations (Golledge, Gould), stratégies d'homo economicus, inégales propensions à accepter la nouveauté (Hägerstrand), positions dans des dispositifs de pouvoir (Harvey).

Cette question passionnante est difficile ! Aujourd'hui encore, lorsqu'il s'agit de comprendre et de formaliser l'émergence d'entités géographiques collectives (des territoires, des réseaux, des systèmes de villes), la théorie ne permet que rarement de relier explicitement le niveau d'action et de représentation des individus et celui des objets géographiques qu'ils construisent, dès que ceux-ci ont une certaine ampleur. On se contente donc souvent de travailler sur des entités agrégées, entre lesquelles existent des régularités empiriques fortes, qui justifient qu'on s'interroge : comment la diversité des intentions, des préférences et des actions, se laisse-t-elle enfermer dans cette dynamique collective si cohérente ? Cela n'implique pas que l'on prenne définitivement parti pour le holisme (Valade, 2001) contre 
l'individualisme méthodologique (Boudon), mais que l'on garde l'un et l'autre en mémoire comme garde-fou de l'autre.

Pour soutenir cette entreprise, la référence aux seuls travaux des géographes n'aurait pas été suffisante, parce que peu d'entre eux encore avaient pris pour objet l'étude de l'évolution des entités géographiques, dans une démarche comparative. Les pionniers (tels D. Janelle ou E. Juillard) n'avaient que des méthodes assez rudimentaires à leur disposition. Il fallait donc constituer des bases de données, et chercher des instruments pour leur analyse. Assez naturellement, c'est l'ensemble des réflexions conduites autour de la dynamique des systèmes qui, à partir des outils qui en étaient issus, offraient les perspectives les plus prometteuses.

\section{De l'analyse des systèmes à l'auto-organisation}

Certains confondraient volontiers le systémisme avec l'esprit de système...quand ce n'est pas avec une espèce de croyance, de type sectaire! L'ambition globalisante des tenants d'une "théorie générale des systèmes » (Bertalanffy, 1969), comme la généralité éclectique et cultivée de certains passeurs (Morin, 1977) ou encore l'enthousiasme prophétique de certains philosophes (Le Moigne, 1983) tantôt séduisent et tantôt laissent en partie sceptique. Outre les réunions organisées par l'AFCET (Association Française pour la Cybernétique Economique et Technique), animées notamment par Bernard Paulré (1985), deux conférences internationales ont jalonné pour moi l'entrée dans un univers de références systémiques : la première organisée à Boston au MIT en 1981, encore très largement dominée par l'analyse de systèmes de type Forrester, et la seconde à Bruxelles en 1982 (AFCET, SOGESCI, 1982) déjà largement consacrée aux théories de l'auto-organisation. Je n'ai découvert que par la suite d'autres approches, notamment celles développées dans le magnifique compte-rendu d'un colloque de Cerisy par Paul Dumouchel et Jean-Pierre Dupuy (1983) et dont bien des interrogations demeurent d'actualité. Pour l'anecdote, c'est lors d'une conférence organisée à Créteil en 1982 sur l'entropie, que j'ai rencontré P. Allen et découvert une formalisation plus générale des observations que nous avions pu faire sur les villes.

Les vingt dernières années ont considérablement fait évoluer le contenu de la référence systémique. Que l'on se souvienne des premières représentations d'un système analysé «à la Forrester » (1964), relativement bien isolé de son environnement, constitué d'éléments peu nombreux en interaction les uns avec les autres, tels que leurs relations à l'intérieur du système soient plus intenses ou plus déterminantes que celles les liant à l'environnement du système, et « organisé pour répondre à un but», selon la définition qu'en donnait Joël de Rosnay (1975).

Au moins cette phase «cybernétique » de la référence systémique a-t-elle permis de mettre au centre des recherches, non plus les entités mais leurs relations, et de bien différencier la simple mesure des évolutions (une cinétique) de la dynamique (incluant une interprétation des facteurs contrôlant cette évolution). Cette première acception du systémisme valorisait aussi l'idée que « le tout est plus que la somme des parties », c'est-à-dire que le comportement du système ne se déduisait pas simplement de celui de ses composantes. J. Forrester insistait sur des «comportements contre-intuitifs» invalidant les bonnes intentions des politiques urbaines, tandis que R. Boudon (1984) mettait en évidence les « effets pervers » de bien des constructions sociales.

Cette idée devient centrale avec les théories de l'auto-organisation, en général issues de la physique, qui considèrent des systèmes à plusieurs niveaux (généralement deux) : le niveau 
microscopique constitué d'un très grand nombre d'éléments en interaction, et le niveau macroscopique où apparaissent des phénomènes collectifs non directement prévisibles à partir de la connaissance des états des éléments microscopiques. Théorie des «structures dissipatives » en chimie (Prigogine, 1979) ou de la «synergétique » issue de la physique des lasers (Haken, 1977) suggèrent des mécanismes interactifs tels que les changements de l'état des particules du système deviennent corrélés entre eux sur de grandes distances (relativement à leur propre taille) et s'auto-organisent selon des configurations macroscopiques.

Surtout, la théorie prévoit que plusieurs configurations sont possibles pour un état du système à l'échelon macroscopique à partir d'une même description des états microscopiques. La dynamique de ces systèmes combine en effet des trajectoires stables et des moments d'instabilité, durant lesquels l'état macroscopique du système peut évoluer vers différentes formes d'organisation, de manière imprévisible, selon des bifurcations. Elle inclut des hypothèses d'irréversibilité du temps, d'imprévisibilité du futur et d'unicité des trajectoires « historiques » de chaque système, faites d'une succession originale de bifurcations. Voilà des propositions qui deviennent conciliables avec l'univers des sciences sociales ! Même si, bien entendu, ces idées n'ont pas pour elles l'intérêt de la nouveauté : depuis que l'on réfléchit sur les sociétés, il n'est pas arrivé que l'on se baignât deux fois dans le même fleuve ! En fait, si ces idées sont séduisantes, c'est qu'elles sont assorties de modèles mathématiques, qui simulent ces processus, et qui sont capables d'engendrer avec les mêmes équations une très grande diversité de formes et d'évolutions. Non seulement leurs hypothèses sont moins réductrices que celles des modèles déterministes ou linéaires, mais en outre ils permettent de se livrer avec meilleure conscience à l'ascèse du test !

Pour la géographie, cette conception présente au moins trois intérêts. Le premier est de permettre le dépassement de l'opposition entre l'idiographique et le nomothétique, qui était un des moteurs de son ambition théorique le plus en rupture avec sa tradition disciplinaire, depuis les années 1970. En effet, la théorie de l'auto-organisation fait comprendre comment des processus généraux identiques sont susceptibles de produire des effets et des structures différents en fonction des conditions initiales et des valeurs des paramètres ${ }^{1}$ qui contrôlent les interactions et leur évolution. La liberté de choix des acteurs, les préférences culturelles ne sont plus exclues de l'explication pour leur incompatibilité avec la production de régularités par des processus généraux. Le second intérêt est d'inviter à repenser la diversité géographique, non plus comme le produit d'une combinatoire contingente autorisant toutes les associations possibles (par exemple de structures élémentaires), mais comme le produit de certains processus fonctionnant sous certaines contraintes et selon diverses temporalités (Durand-Dastès, 1996), qu'il s'agit de mesurer et de hiérarchiser. Les particularités des entités géographiques sont explicables par une succession originale de trajectoires stables et de bifurcations, selon une évolution qui procède par sauts, et qui entraîne le système vers des attracteurs distincts. Les structures observées à un moment donné intègrent cette « histoire» particulière produite par une dynamique générique, et donc toutes les associations qualitatives de propriétés ne sont pas également probables.

Ainsi, une troisième source de satisfaction intellectuelle réside dans une forme de libération par rapport au caractère surplombant et inéluctablement premier de l'histoire dans l'explication des entités géographiques: au lieu que celle-ci ne prenne nécessairement la forme du récit biographique, à faire remonter toujours plus loin du côté des origines et

\footnotetext{
${ }^{1}$ Un exemple facile est celui de la configuration sociale centre-périphérie de certaines villes nord-américaines et de nombreuses villes européennes, de forme opposée en dépit de la similitude de certaines des contraintes et des processus sociaux qui les déterminent.
} 
toujours à actualiser en fonction des transformations du présent, apparaît une nouvelle forme d'intelligibilité de l'évolution des lieux, qui permet de les caractériser en tant que réalisation advenue, parmi toute une collection de résultats possibles, dans l'évolution d'un système dynamique. Refaire l'histoire, ou l'inventer? La simulation par les modèles permet en quelque sorte de sortir la géographie de son rôle de fille de l'histoire, condamnée à recourir au fil d'Ariane du récit biographique pour trouver du sens dans le labyrinthe causal des interactions sociales, écologiques et spatiales, en lui substituant une explication, certes réduite, "normalisée », sous forme d'une trajectoire dynamique dans un processus évolutif générique.

Les expériences de simulation menées avec ce type de modèles sont encore trop peu nombreuses et trop peu confrontées entre elles pour juger de la pertinence et de la qualité de cette contribution explicative. Sans doute une meilleure évaluation sera-t-elle possible en fonction de l'aide qu'ils peuvent apporter à la décision territoriale, tout comme à l'arsenal des méthodes de prévision. L'apport essentiel réside dans la mise en relation explicite de configurations géographiques, de structures spatiales, avec des processus générateurs agissant à d'autres échelles.

\section{De l'auto-organisation à la complexité}

Les théories de la complexité aujourd'hui mettent l'accent sur une notion plus difficile, celle de «structure émergente ». Déjà présente en physique avec le concept d'auto-organisation, cette idée, issue principalement de l'étude du vivant, semble ajouter une connotation de création, d'apparition de la nouveauté. Par rapport au système préexistant, l'émergence peut consister dans la création d'une nouvelle catégorie d'éléments, ou d'attributs, ou dans l'apparition d'une nouvelle structure, voire d'un nouveau système, ou dans l'invention de nouvelles règles par les protagonistes d'un jeu de simulation. Ainsi, dans les références actuelles, on parle moins d'auto-organisation et plus d'émergence, ce qui se définit strictement de la même façon en termes de relation entre niveaux dans le système, mais l'accent est désormais mis sur la nouveauté, voire la "surprise » de l'observateur (qui se trouve donc impliqué dans la conception du système), et peut s'exprimer en termes de règles au niveau des interactions entre agents (qui deviennent des acteurs intelligents et eux-mêmes adaptatifs, non plus passifs comme les particules des structures dissipatives ou de la synergétique).

Les risques de l'analogie entre les systèmes vivants et les systèmes sociaux sont bien connus. La sociobiologie est sans doute la plus critiquée de ces tentatives, parce qu'en naturalisant les rapports sociaux, on pourrait laisser entendre que, puisque ces derniers sont aussi " naturels », il serait illusoire d'essayer de les transformer. Or, cette représentation-là est en train de changer : avec les biotechnologies, la société fait irruption dans le champ de l'évolution du vivant, le «naturel» devient aussi transformable. Cela ne va pas sans poser des problèmes éthiques. Mais alors sur quels arguments vont pouvoir s'appuyer tous ceux qui sont réticents à l'idée d'une science sociale reposant sur les mêmes principes et les mêmes méthodes que les sciences du vivant (Passeron, 1991), et qui récusent les éléments d'ingénierie sociale qui en dériveraient?

La théorie actuelle de l'évolution a certes acquis une plus grande compatibilité avec les conceptions des sciences sociales (fin du finalisme, place dévolue à la contingence, notion d'adaptation sans optimalité), elle admet aussi que l'évolution puisse en partie dépendre des interactions avec l'environnement (Mayr, 1989, Prochiantz, 2001, Jeannerod, 2002). Mais, 
outre que les conceptions se modifient très vite dans ce champ des sciences d'observation, sous l'effet des progrès des techniques et sous le contrôle des méthodes expérimentales, s'inspirer des théories évolutionnistes ne serait sans doute pas la bonne réponse pour continuer à construire une théorie géographique. Le risque est en effet de passer à côté de deux processus essentiels : celui de l'invention, non plus aléatoire ou à la rigueur guidée par l'environnement, comme les mutations du vivant, mais de plus en plus consciente, recherchée, anticipée dans l'émulation par rapport à d'autres individus ou groupes sociaux, et moteur apparemment sans fin qui débloque les verrous successifs à la maîtrise humaine de l'utilisation des ressources planétaires. L'autre spécificité des systèmes sociaux est l'intervention de la cognition, qui injecte dans le système des stratégies, certes plus ou moins bien informées, mais qui tiennent compte d'une représentation de l'état du système pour tenter d'agir dessus (voir par exemple les prophéties à visée auto-réalisatrice du marketing urbain). Dans les diverses théories du lien social (qu'elles reposent sur l'imposition violente d'un pouvoir, ou sur un consensus culturel, ou sur la rivalité mimétique) on démontre que l'effet des représentations de ces théories qui filtrent dans la société ne sont pas neutres vis-àvis de son évolution (conscience de classe, prophéties auto-réalisatrices). Tout se passe comme si, collectivement, l'humanité guidait en partie son évolution sociale, et peut-être bientôt biologique, à partir des représentations qu'elle s'en fait.

Dans ces investigations, une des contributions de la géographie peut être d'aider à mieux comprendre l'intervention de niveaux intermédiaires entre les représentations individuelles et la formation ou le maintien de grands systèmes sociaux. Entre l'individu et le monde, à côté (ou plutôt en conjonction) des groupes sociaux, des institutions, étudiées par d'autres disciplines comme la sociologie ou la science politique, la géographie signale l'importance, pour la structuration et l'évolution de ces systèmes, de niveaux d'organisation localisés et spatialisés, villes, régions, réseaux, territoires...La médiation des relations entre les individus et la société par des lieux différenciés, articulés en niveaux d'échelle, devient, tout autant que l'analyse de l'évolution de ces entités, un programme (Berthelot, 2001) spécifique à la géographie dans les recherches sur la complexité en sciences humaines et sociales.

Les théories actuelles des systèmes complexes s'efforcent de trouver des interprétations globalisantes pour rendre compte de l'émergence de la nouveauté, dans des champs aussi divers que l'économie cognitive (Kirman,1993), la psychologie sociale, ou les systèmes homme-machine (Hubermann, 1988). La géographie pourrait être tentée d'aller emprunter de nouveaux modèles à l'une ou l'autre de ces disciplines. Pourtant, tout en gardant une attention soutenue à ces expérimentations en cours, il me semble que c'est désormais dans son fonds propre de références que la géographie peut trouver matière à construire des éléments théoriques pertinents. Parmi les instruments mis à sa disposition, les modèles de systèmes multi-agents, couplés à des SIG, sont en effet de nature à permettre une formalisation contrôlée de certaines des hypothèses qu'elle avance depuis plusieurs décennies sans avoir pu complètement les tester : sur le rôle des acteurs dans les transformations des territoires, sur la place que prennent les représentations dans ces processus, sur les mécanismes de l'adaptation et de la spéciation des entités géographiques.

Le statut épistémologique de l'émergence dans les modèles actuellement réalisables demeure cependant ambigu. Dans le monde réel, que ce soit celui du vivant ou celui du social, de la nouveauté est produite, elle émerge véritablement, à partir de ce qui existe. Dans la transcription que nous en faisons dans les ordinateurs, les phénomènes d'émergence que nous savons simuler pour le moment, qui produisent un effet de surprise pour l'observateur, sont le plus souvent liés à la difficulté de celui-ci à concevoir le résultat du jeu des règles qu'il a lui- 
même introduites dans le modèle. Seule la rapidité de calcul de la machine, en permettant de dépasser les limitations de l'esprit humain, produit un effet de surprise, en réalisant une sorte de compression du temps. Mais la simulation de l'émergence d'une véritable nouveauté au moyen de modèles reste très difficile. Par exemple, dans un modèle proposé par P. Allen et $\mathrm{J}$. McGlade, les «innovations» d'acteurs non optimisateurs censés inventer une fonction productive ou sociale nouvelle sont en fait représentées dans le modèle par des sauts de leur position dans le graphe de l'espace des phases, qui pourraient se traduire comme l'exploration géographique d'un continent éloigné ou la colonisation d'un nouveau territoire ! Mais c'est peut-être en recherchant ce déplacement des métaphores d'une discipline à l'autre que, de temps en temps, peut émerger ce qui constitue véritablement une innovation scientifique.

Il reste qu'au cours de cette évolution scientifique, on a relâché beaucoup des exigences qui rendaient réductrice et contestable l'opération consistant à isoler par la pensée, dans le réel social, des observations et des propriétés, pour un faire un système relativement isolé de son environnement. Cela ne signifie pas que cette opération ne reste pas la plus délicate de toutes (Batty, Torrens, 2002). Elle est cependant facilitée par cette propension de ce que nous appelons des «systèmes » à survivre, à s'auto-entretenir, à être résilients, à s'adapter, à s'auto-transformer pour durer. Que cela résulte de la tendance croissante à l'isolement, au fonctionnement intégré et autonome, voire auto-référent, comme le suggère Luhman (1999) à propos des systèmes institutionnels dans les sociétés occidentales développées, ou bien que cela soit associé plus étroitement à nos filtres cognitifs d'observation du réel, que cela ressorte d'une position constructiviste ou d'une affirmation réaliste (comme celle de Prigogine associant l'existence d'entités observables à une dynamique discrète, qui "saute » d'une trajectoire à l'autre sans emprunter toutes les configurations intermédiaires imaginables), au fond peu importe. Le paradigme est porteur, puisqu'il nous invite aussi à dépasser les apories de l'opposition entre holisme et individualisme.

«Ce qu'on ne sait pas dire, il faut le taire » disait Wittgenstein. Ce fondement d'un type de communication scientifique (ne se poser que les questions qu'on peut résoudre, n'avancer que ce que l'on a empiriquement testé, signaler quand on s'évade et qu'on commence à divaguer) n'est lui-même pas scientifique au sens des sciences sociales, puisqu'il est bien réfuté par l'expérience! Qu'on nous permette de l'invoquer cependant pour ne pas avoir à choisir entre deux définitions de la complexité, réaliste : «la complexité est un ordre dont on ne connaît pas le code» (Atlan, 1979, p .78), ou constructiviste «la complexité est le nombre d'interprétations non équivalentes qu'un observateur peut se faire d'un système » (Livet, 1983).

\section{Conclusion}

Sans doute l'une des perspectives les plus prometteuses de cette démarche appuyée sur le comparatisme de la dynamique des systèmes est de pouvoir expliquer des objets géographiques (territoires, villes, régions, réseaux, systèmes de villes) non plus par l'aboutissement d'un récit biographique mais comme l'une des issues possibles d'un ensemble de processus interactifs complexes. Il ne s'agit pas de remplacer des processus géographiques (écologiques et socio-spatiaux) par des processus explicatifs qui seraient pertinents pour d'autre systèmes, mais bien d'imaginer que l'explication des évolutions observées relève pour une part (qu'il s'agit de déterminer) de dynamiques évolutives plus générales, communes à un certain nombre de systèmes, et que l'on peut abstraire dans des modèles, mathématiques ou informatiques. 
Une telle démarche a l'intérêt pour les sciences sociales de permettre de substituer à la linéarité du récit de l'histoire événementielle le résultat plus ou moins aléatoire de dynamiques compétitives susceptibles d'être modélisées, et de décrire les systèmes viables observés comme la réalisation de trajectoires particulières parmi un ensemble de possibles, sous des contraintes identifiables.

Je retiendrais ainsi volontiers de mes expériences d'échanges intellectuels et de transferts de modèles une disposition favorable à la métaphore et à l'analogie, sous le contrôle vigilant de ce qui fait l'identité disciplinaire : chercher des transpositions d'une science à l'autre permet d'abord de mieux réfléchir à ce qui fait la spécificité des objets dont s'occupe chacune. Un bon exemple en serait donné aujourd'hui par les sciences cognitives, les allers retours entre les représentations des ordinateurs et celles du cerveau ayant produit beaucoup de fantasmes et d'illusions mais permis au passage des investigations mieux centrées, et de nouvelles applications.

Les similitudes de morphologie, de comportement ou d'évolution entre les systèmes, en somme des manifestations extérieures de leur activité, lorsqu'elles sont comparables, signifient-elles une similitude des processus qui les produisent? Là est la question. Du moins invitent-elles à étudier des descriptions nouvelles susceptibles d'éveiller d'autres hypothèses explicatives. D'où l'utilité des emprunts en termes de méthodes d'analyse et de mesure des morphologies et des dynamiques, les références instrumentales ayant pour moi toujours prévalu sur les analogies interprétatives.

On commence à peine à comprendre le rôle de la diversité dans une dynamique. Mais il n'est peut-être pas trop ambitieux d'envisager pour la géographie, comme sa contribution, non seulement aux sciences, mais encore à l'éthique (Smith, 2000), une théorie qui montrerait comment la diversité planétaire et mondiale, d'origine « naturelle » et construite, constitue le moteur historiquement le plus sûr du changement social, en incitant d'abord à aller voir ailleurs, puis à mettre en réseau, en suscitant toujours l'émulation, même si prévaut souvent la prédation... Encore cette vague intuition doit-elle être formalisée et modélisée pour emporter la conviction et devenir une référence en dehors de la géographie...

\section{Références}

Abler R. Adams J., Gould P., 1972, Spatial organization, the geographer's view of the world. London, Prentice Hall.

AFCET-SOGESCI, 1982, Des sciences physiques aux sciences sociales. Bruxelles, 7eme conférence internationale de dynamique des systèmes, 2 vol.

Allen P. 1997, Cities and regions as self-organizing systems: models of complexity. Amsterdam, Gordon and Breach.

Atlan H., 1979, Entre le cristal et la fumée. Paris, Seuil.

Batty M., Torrens P., 2001, Modelling complexity. The limits to prediction. Cybergeo, 201, $24 \mathrm{p}$.

Berthelot J.-M., (dir.) 2001, Epistémologie des sciences sociales. Paris, PUF. 
Boudon R. 1984, La place du désordre. Paris, PUF.

Claval P. 1979, La nouvelle géographie. Paris, PUF, coll. Que-sais-je.

Dumouchel P. et Dupuy J.P. 1983, L'auto-organisation, de la physique au politique. Paris, Seuil, colloque de Cerisy.

Durand-Dastès F. 1996, La trace des temps, in Archaeomedes, Des oppida aux métropoles, Paris, Anthropos, 45-71.

Forrester J. 1964, Urban dynamics. Boston, MIT Press.

Hubermann B. A. (ed.), 1988, The ecology of computation. North Holland, Elsevier.

Haken H., 1977, Synergetics, an introduction. Berlin, Springer.

Jeannerod M. 2002, La nature de l'esprit. Paris, Odile Jacob.

Kirman A. 1993, L'hypothèse de l'individu représentatif : une analyse critique. Problèmes économiques, $\mathrm{n}^{\circ} 2325,5-14$.

Lacoste Y. 1976, La géographie, ça sert d'abord à faire la guerre.

Le Moigne J.-L., 1983, Science de l'autonomie et autonomie de la science, in Dumouchel P., Dupuy J.-P. (eds) op.cité, 521-536.

Livet P. 1983, La fascination de l'auto-organisation, in Dumouchel P., Dupuy J.-P. (eds) op.cité, 165-171.

Luhman N. 1999, Politique et complexité. Paris, Ed. du Cerf.

Mayr E. 1989, Histoire de la biologie, Paris, Fayard, 2 vol.

Morin E., 1977, La méthode. Paris, Seuil, 3 vol.

Passeron J.-C. 1991, Le raisonnement sociologique. Paris, Nathan.

Paulré B., 1985, La causalité en économie. Presses Universitaires de Lyon.

Pinchemel Ph. et G., 1988, La face de la terre. Paris, Colin.

Prigogine I., Stengers I., 1979, La nouvelle alliance, Paris, Gallimard.

Prochiantz A. 2001, La machine-esprit. Paris, Odile Jacob.

Pumain D. 1997, Vers une théorie évolutive des villes. L'Espace Géographique, 2, 119-134.

Pumain D. 1998, La géographie saurait-elle inventer le futur? Revue européenne des sciences sociales, 110, 53-69. 
Pumain D. 2001 Approche systémique et causalité: à propos des systèmes de villes. Vanves, CNED, N6106 T 03, 405, p.65-103.

Pumain D. Robic M.C. 1996, Théoriser la ville, in Derycke P.H. Huriot J.M. Pumain D. (eds), Penser la ville, théories et modèles. Paris, Anthropos, chap. 4, 107-161.

Pumain D. Robic M.-C. 2002, Le rôle des mathématiques dans une « révolution » théorique et quantitative : la géographie française depuis les années 1970. Revue d'histoire des sciences humaines, 6, 123-144.

Racine J.B., Reymond H., 1973, L'analyse quantitative en géographie. Paris, PUF.

de Rosnay J., 1975, Le macroscope, Paris, Seuil.

Rosser J.B., 1991, From catastrophe to chaos : a general theory of economic discontinuities. Dordrecht, Kluwer.

Smith D. 2000, Moral Geographies. Ethics in a World of Difference. Edinburgh University Press.

Valade B. 2001, De l'explication dans les sciences sociales: holisme et individualisme, in Berthelot J.-M., (dir.), Epistémologie des sciences sociales, Paris, PUF, 357-405. 